\section{Journal of \\ Epilepsy and \\ Clinical \\ Neurophysiology}

JEpilepsy Clin Neurophysiol 2008; 14(4):162-170

\title{
Aspectos Epidemiológicos das Comorbidades Psiquiátricas em Epilepsia*
}

\author{
Marleide da Mota Gomes \\ Instituto de Neurologia Deolindo Couto - Universidade Federal do Rio de Janeiro (UFRJ)
}

\begin{abstract}
RESUMO
Introdução: Várias comorbidades psiquiátricas à epilepsia são prevalentes e podem reduzir a qualidade de vida ligada à saúde (QVLS) das pessoas com epilepsia (PE). Objetivos: Abordar a magnitude do problema, distribuição dos transtornos psiquiátricos mais associados, segundo a distribuição por idade e gênero. Reconhecer: os fatores de risco para essas comorbidades e a sua classificação/diagnóstico; os índices de multimorbidade de potencial uso em epileptologia e a sua aplicabilidade na avaliação da QVLS; repercussão na prática clínica da abordagem das mencionadas comorbidades. Métodos: Revisão narrativa sobre comorbidade psiquiátrica, principalmente com artigos dos últimos 10 anos de base populacional. Resultados: Os transtornos psiquiátricos são mais prevalentes nas PE do que na população geral, sendo os transtornos do humor, ansiedade, psicoses, esquizofrenia, transtornos de personalidade e dependência alcoólica os mais comuns. Vários deles são mais prevalentes em qualquer faixa etária das PE do que na população geral, e mais nas mulheres do que nos homens, e tendem a repercutir negativamente no seu nível sócio-econômico. $\mathrm{O}$ diagnóstico dessas comorbidades é limitado: não costuma haver distinção entre transtornos psiquiátricos gerais e os especificamente relacionados à epilepsia. Conclusão: Os transtornos psiquiátricos são comuns nas PE, especialmente os do humor, mas as taxas são diversas assim como as metodologias empregues, e há necessidade de sua melhor abordagem para melhor controle clínico dos pacientes, melhoria da sua QVLS e redução de custos dos cuidados à saúde.
\end{abstract}

Unitermos: Epilepsia, comorbidade, psicopatologia, qualidade de vida ligada à saúde, índice de multimorbidade.

\begin{abstract}
Epidemiological issues of the psychiatric comorbidities in epilepsy

Introduction: Several psychiatric comorbidities linked to epilepsy are prevalent, and they can reduce the people with epilepsy (PE) health-related quality of life (HRQOL). Objectives: To approach the magnitude of the psychiatric comorbidities, their distribution, according to the distribution by age and gender. To recognize: the risk factors for them, and their classification/diagnosis; the indexes of multimorbididity of potential use in epileptology, and their applicability in HRQOL; repercussion in the clinical practice of the approach of the mentioned comorbidities. Methods: Narrative revision on psychiatric comorbidities, mainly based on population based studies published in the last 10 years. Results: The psychiatric disorders are more prevalent in the PE than in the general population, being the humor disorder, anxiety, psychoses, schizophrenia, personality disorder, and alcoholic dependence the most common. Several of them are more prevalent in any age group of the PE than in the general population, and more in the women than in the men, and they tend to echo negatively in their socioeconomic level. The diagnosis of these comorbidities is limited: it is not common the distinction between general psychiatric disorders and those related to the epilepsy. Conclusions: The psychiatric disorders are common in the PE, mainly of humor, but the rates are diversified as well as the used methodologies, and there is need of better approach of them to the patients' better clinical control, improvement of their HRQOL and reduction of health care costs.
\end{abstract}

Key words: Epilepsy, comorbidity, psychopathology, health-related quality of life, multimorbidity index.

\footnotetext{
* Trabalho apresentado parcialmente no XXXII Congresso da Liga Brasileira de Epilepsia, na mesa-redonda Epidemiologia e qualidade de vida, realizado em junho de 2008, em Campinas.

Received June 17, 2008; accepted Sept. 30, 2008
} 


\section{INTRODUÇÃO}

O termo comorbidade foi introduzido em medicina por Alvan Feinstein (1970) com a seguinte definição: "qualquer entidade adicional distinta que existiu ou pode acontecer durante o curso clínico de um paciente que tem a doença índice em estudo". ${ }^{1}$ Hoje essa designação vem assumindo outras conotações e para melhor delimitação Van den Akker et al., 1996, apud Fortin et al., 2004,1 "reservou o termo 'multimorbidade' para descrever a coocorrência de duas ou mais condições crônicas. Eles também propuseram alguns qualificadores para melhor classificar o tipo de multimorbidade (simples, associativa e causal)". Assim, atualmente, pode-se considerar comorbidade, na dependência do critério conceitual usado, uma condição médica em um paciente que causa, é causada por, ou é relacionado ou simplesmente coexiste à condição índice. Essas relações podem ser bem complexas na epilepsia, pois as alterações psiquiátricas podem ser periictais (pré, ictal ou pós) ou interictais. ${ }^{2-4}$ Há de se ressaltar que com o aumento da sobrevida de pessoas com doenças previamente fatais e o envelhecimento da população, uma proporção crescente de pacientes vem se apresentando com múltiplas condições médicas coexistentes. ${ }^{1}$ Assim, em condições crônicas, como a epilepsia, a coexistência de mais de uma doença em um paciente é a regra em lugar de exceção. Os homens e mulheres com epilepsia têm aumento da ocorrência de transtornos cerebrovascular e cardiovasculares, gastrointestinal, pulmonares, além de enxaqueca, demência, fadiga crônica, transtornos do humor, ansiedade e transtornos de personalidade. ${ }^{5-8}$ Impressiona o aumento da prevalência de enxaqueca em pacientes com epilepsia (PE) que pode ser uma associação espúria e/ou revelação de mecanismos fisiopatogênicos similares: ${ }^{7}$ raciocínio equivalente ao da relação entre comorbidades psiquiátricas e a epilepsia. Em estudos que avaliam o número de comorbidades em transtornos neurológicas, como o de Nuyen et al., 2006, 8 o número de categorias é: esclerose múltipla, 3; doença de Parkinson/parkinsonismo, 4; demência, 5; epilepsia, 11; enxaqueca, 11; AVC, 21; depressão, 26. Assim, pela sua relevância, o tema tem sido crescentemente abordado, principalmente nos últimos anos, como podemos verificar no levantamento feito (Figura 1), no banco bibliográfico PUBMED, a partir dos descritores comorbidit*[title/abstract] AND epilepsy[title/abstract], no período de 1986 a 2008 (1ํartigo registrado em 1986, no total de 284. Pesquisa concluída em 28.05.2008). Particularmente, este artigo é composto como revisão narrativa e com artigos relacionados predominantemente à comorbidade psiquiátrica e à epilepsia como doença índice, nos últimos 10 anos e tendo como objetivo levantar questões sobre a magnitude do problema, distribuição dos transtornos psiquiátricos mais associados, principalmente a partir de estudos populacionais, segundo a distribuição etária e de gênero. Abordam-se também os fatores de risco para comorbidades psiquiátricas, principais instrumentos usados para o diagnóstico das comorbidades psiquiátricas e dificuldades diagnósticas, os índices de multicomorbidade de potencial uso em epileptologia e, alguns, na avaliação da qualidade de vida ligada à saúde (QVLS).

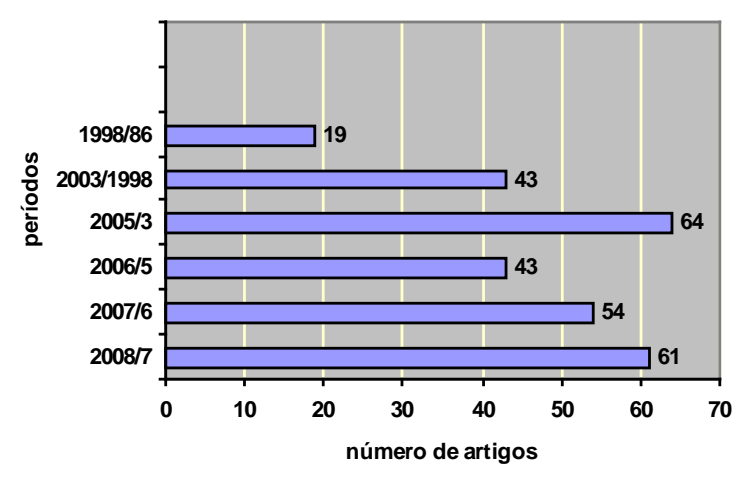

Figura. Comorbidade e epilepsia no Pubmed (nos últimos 3 anos, 55,6\% de toda a produção)

\section{DISTRIBUIÇÃO POPULACIONAL}

As prevalências de transtornos psiquiátricas a partir de populações selecionadas, por exemplo, de PE do lobo temporal e/ou farmacorresistente, indicam a relevância destes transtornos, ${ }^{5}$ mas, essas prevalências podem ser superestimadas. Há variação extensa nas taxas de prevalência obtidas por conta da diversidade do tamanho e características das amostras examinadas, métodos de captação, critérios classificatórios das comorbidades psiquiátricas (e mesmo da epilepsia), prazo de avaliação (duração do seguimento) e desenho de estudo, por exemplo. Estudos de países desenvolvidos, envolvendo metodologia e amostras com tipos diversos de epilepsia, constantemente informam uma prevalência mais alta de condições psiquiátricas em PE do que na população geral ou outros grupos de controle neurológico ou pessoas com transtornos não neurológicos crônicos, cujas natureza e prevalência variam com idade e sexo, apesar de ainda haver carência destes estudos em grupos socio-econômicos diversos e populações diversas, como as brasileiras. As comorbidades psiquiátricas mais frequentes informadas em PE incluem psicoses, neuroses, transtornos do humor (DSM-IV eixo I), transtornos de personalidade (DSM-IV eixo II), e problemas comportamentais, segundo Gaitatzis et al., $2004^{3}$ e Gaitatzis et al, 2004, ${ }^{5}$ sendo que a "depressão" é a comorbidade psiquiátrica mais comumente definida em PE. ${ }^{5,6,9}$ Gaitatzis et al., 2004, ${ }^{3}$ conduziram uma revisão não sistemática sobre a prevalência de transtornos psiquiátricas em pessoas com epilepsia. Eles concluíram que 6\% das PE na população geral parecem sofrer de uma transtorno 
psiquiátrico, mas este percentual sobe para $20-50 \%$ em populações mais selecionadas.

O estudo populacional mais recentemente publicado de prevalência de comorbidade psiquiátrica em epilepsia é o de Tellez-Zenteno et al., 2007. ${ }^{6}$ Os autores usaram os dados do Canadian Community Health Survey (CCHS) e compararam a prevalência desses transtornos em pessoas sem epilepsia (36731) com as com epilepsia (253), de 15 ou mais anos. A entrevista de saúde mental foi baseada no World Mental Health Survey Initiative Version of the World Health Organization Composite International Diagnostic Interview (WMH-CIDI). O CIDI é uma entrevista diagnóstica estruturada destinada a determinar síndromes psiquiátricas de acordo com o DSM-III-R, eixo I. Swinkels et al., 2001, ${ }^{10}$ lembram que deveria ser reconhecido que o CIDI não é validado para PE. Na verdade, em geral os estudos não são desenhados para investigar a psicopatologia específica da epilepsia, como psicose pós-ictal. No estudo de Tellez-Zenteno et al., 2007, ${ }^{6}$ sobre a prevalência de comorbidades psiquiátricas em epilepsia, entrevistadores de campo treinados aplicaram a entrevista com o uso de computador. Os seguintes transtornos foram avaliados: transtorno depressivo maior, transtorno do humor, transtorno de ansiedade, transtorno bipolar, fobia social, agorafobia, transtorno do pânico, dependência de substância e ideação suicida. Os algoritmos diagnósticos seguiram os critérios do DSM-IV, com a exceção da exigência de duração para um episódio maníaco. No CCHS, somente se perguntou se os sintomas maníacos tinham durado vários dias ou mais tempo, mas a duração de sete dias é requerida pelo DSM-IV quando não houve necessidade de internação hospitalar, como informaram os autores. Nessa análise, os autores diferenciaram transtorno depressivo maior do transtorno bipolar, identificando os sujeitos com um ou mais episódios maníacos ao longo da vida de acordo com o CIDI. Não foram avaliados dois transtornos, distimia e esquizofrenia usando um módulo do CIDI, mas ao invés, foram obtidos, a partir da informação dos diagnósticos de profissionais, como foi feito com o diagnóstico de epilepsia (autodeclarado). A presença de epilepsia foi questionada por um entrevistador que perguntou diretamente ao sujeito "Se tem epilepsia diagnosticada por um profissional de saúde?". Aqueles que responderam "sim" foram considerados como tendo epilepsia. Um outro problema foi o da taxa de prevalência de algumas comorbidades psiquiátricas como transtorno bipolar, esquizofrenia, transtornos de personalidade, psicose, e dependência de substância não puderam ser calculadas por causa do tamanho pequeno da amostra. No entanto, a qualidade maior desse estudo é o método de captação feito pelo CIDI que foi validado para averiguar condições psiquiátricas em populações grandes. No estudo de TellezZenteno et al., 2007, ${ }^{6}$ a prevalência de transtornos de saúde mental em pessoas sem e com epilepsia em 12 meses foi
(IC de 95\%): transtorno do humor, 5,2 (4,9-5,5) vs. 14,1 $(7,0-21,1)$; transtorno de ansiedade, $4,6(4,3-4,9)$ vs. 12,8 $(6,0-19,7)$; transtorno do humor/ansiedade, 8,0 (7,6-8,5) vs. 19,9 (12,3-27,4); transtorno do pânico/agorafobia, 2,0 $(1,8-2,2)$ vs. 5,6 (1,9-9,2); qualquer transtorno de saúde mental, $10,9(10,4-11,3)$ vs. 23,5 (15,8-31,2). A ideação suicida (prevalência ao longo da vida) é de 13,3 vs. $25 \%$, nos sem e com epilepsia, respectivamente. De acordo com a estimativa feita pelos autores do trabalho canadense, problemas psiquiátricos variam com a idade em um padrão que é diferente da população geral: 1 - a prevalência ao longo da vida de transtornos depressivos maiores declinam com a idade em mulheres com e sem epilepsia, mas permanece estável em homens, mas é globalmente mais alta em pessoas com epilepsia; 2 - a prevalência de transtorno do pânico e agorafobia em 12 meses só aumentou com a idade em pessoas com epilepsia. Assim, o tipo e a prevalência de comorbidades psiquiátricas com epilepsia é mais alta para qualquer faixa etária, ${ }^{6}$ mas, mais especificamente, Bijl et al., 1997, apud Swikels et al., 2001, ${ }^{10}$ reiteram que os pacientes mais velhos sofrem menos de transtornos psiquiátricos. Isso também está de acordo com Swikels et al., 2001, ${ }^{10} \mathrm{em}$ dados relativos a humor, ansiedade, e transtornos relacionados ao uso de substâncias psicoativas.

A Tabela 1, adaptada de Tellez-Zenteno et al., 2007, ${ }^{6}$ apresenta os resultados de alguns estudos populacionais com controles, nos últimos 10 anos. 5,6,9,11,12

Strine et al., 2005,13 conduziram um estudo de 427 PE comparados com 30018 indivíduos baseado no 2002 National Health Interview Survey (NHIS). O NHIS mediu angústia psicológica não específica em um período de 30 dias com o uso da Escala de Kessler. Eles concluíram que as pessoas com $\mathrm{CE}$ tinham significativamente maior probabilidade do que os outros de informar níveis mais baixos de educação, níveis mais altos de desemprego, dor, hipersonia e insônia, e angústia psicológica (por exemplo, sentimentos de tristeza, nervosismo, desesperança e inutilidade). Stefansson et al., 2008, ${ }^{14}$ compararam a prevalência de transtornos psiquiátricos não orgânicos em pacientes de inteligência normal com epilepsia (241, de 16 a 66 anos de idade) com pacientes com outras doenças somáticas (482). O sexo e a idade foram pareados, e ambos grupos recebiam benefícios por incapacidades. Os autores mencionam que os dados, baseados em um estudo recente de prevalência de epilepsia na Islândia, indicam que em torno de metade desta população com epilepsia recebe os mencionados benefícios. Os diagnósticos psiquiátricos baseados nos critérios da CID-9 entre pacientes inválidos com e sem epilepsia foram: 35,3 vs. 29,7; psicoses, 6,2 vs. 2,3; doença neurótica ou transtornos de personalidade, 18,3 vs. 21,0; abuso de álcool e dependência de droga, 5,0 vs. 2,3; outros transtornos não psicóticos 5,8 vs. 4,0. Outra indicação é a de que os pacientes inválidos com 
epilepsia (depois de excluir os pacientes com retardo mental, autismo, e doença mental orgânica dos casos e controles), provavelmente têm mais doença psicótica do que os outros pacientes. Assim, variações significantes na prevalência de condições psiquiátricas em PE existem entre os já mencionados estudos. Eles podem superestimar/ subestimar a prevalência dos transtornos psiquiátricas, em parte devido à falta de entrevistas padronizadas. Lembrar que os vieses de overdiagnosis em epilepsia colaboram em parte com os resultados de maiores taxas de comorbidade psiquiátrica. Isso nos reporta a estudo epidemiológico realizado no Rio de Janeiro que teve a grande preocupação da análise meticulosa de caso a caso. ${ }^{15}$ Nele, houve a demonstração de em que vários sujeitos com suspeita de epilepsia na primeira fase de estudo de porta-a-porta se demonstrou, na segunda fase, tratar-se apenas de eventos não epilépticos, muitos deles transtornos psiquiátricos. Assim, há de se questionar se principalmente os estudos que demonstram altas taxas de epilepsia, mais do que a média usual de 0,5 a 1,0\%, apresentariam indevidas altas taxas de comorbidades psiquiátricas, pois na verdade, o transtorno índice já seria de natureza básica psiquiátrica. Portanto, reiteramos que há a possibilidade de que alguns dos $\mathrm{PE}$ em alguns estudos realmente não sejam epilépticos. Observa-se que nos estudos de Strine et al., 2005, ${ }^{13}$ Kobau et al., 2006, ${ }^{9}$ e Tellez-Zenteno et al., 2007, ${ }^{6}$ por exemplo, o diagnóstico de epilepsia é auto-informado. Por sua vez, a avaliação dos transtornos psiquiátricos também em alguns casos foi feito de forma inespecífica: a partir de sintomatologia de depressão/ansiedade (seis manifestações de angústia) ou autodeclarada, como nos estudos de Strine et al., 2005,13 e Kobau et al., 2006, ${ }^{9}$ respectivamente. Particularmente, as taxas mais altas de transtornos psiquiátricos tendem a advir de registros de médicos generalistas, sendo a razão previamente já apontada uma causa importante (falso positivo). Outro estudo realizado em Tóquio sob a liderança de Matsurura et al., 2003, ${ }^{16}$ todos neuropsiquiatras e epileptólogos, preocupou-se com a prevalência de transtornos psiquiátricos em $398 \mathrm{PE}$ recentemente referidos para nove clínicas neuropsiquiátricas especializadas em epilepsia. As prevalências de transtornos psiquiátricos foram (\%): geral, 24 (23, com retardo mental; 18, transtorno de personalidade); neuróticos, 8; espectro esquizofrênico, 7; afetivos, 1, apenas. Esse último dado bastante estranho ao referido na literatura, poderia ser devido à dificuldade classificatória, segundo os autores, pois essa sintomatologia nos $\mathrm{PE}$ foge aos critérios usuais da CID-10 e DSM-IV. No estudo de Swinkels et al., 2001, ${ }^{10}$ sobre a prevalência de psicopatologia, foram estudados 209 pacientes consecutivamente atendidos em centro psiquiátrico de epilepsia. A prevalência no último ano para os transtornos foi: ansiedade, 25\%; humor, 19\%. Em crianças, os tipos de transtornos psiquiátrico informados são freqüentemente diferentes dos de adultos, e há uma escassez relativa de dados. ${ }^{3}$ No entanto, sabe-se que esses transtornos constituem um óbice significativo às crianças e às suas famílias. ${ }^{12,17}$ Davies et al., 2003,12 realizaram estudo de base populacional: 67 crianças, sendo $42 \mathrm{com}$ epilepsia não complicada e 25 com epilepsia complicada. Os autores encontraram uma taxa de $37 \%$ de transtornos psiquiátricos em crianças com epilepsia, comparativamente a 11\% em crianças diabéticas e $9 \%$ na população geral. Nas crianças com epilepsia, mais especificamente encontraram nas com epilepsia complicada vs. não complicada os seguintes transtornos (\%): qualquer, 56,0 vs. 26.2 ; emocional, 16,0 vs. 16,7 ; conduta, 24,0 vs. 16,7 ; transtorno do deficit de atenção e hiperatividade (TDAH), 12,0 vs. 0; autístico, 16,0 vs. 0.

Tabela 1. Estudos em populações não selecionadas com controles, nos últimos 10 anos: epilepsia e comorbidade psiquiátrica (adaptada de Tellez-Zenteno et al., 2007).

\begin{tabular}{|c|c|c|c|c|c|c|c|c|c|}
\hline \multirow{2}{*}{ Autor } & \multirow{2}{*}{$\begin{array}{l}\text { N/Instrumentos } \\
\text { de avaliação }\end{array}$} & \multirow{2}{*}{ Tipo de população } & \multicolumn{7}{|c|}{ Prevalência de transtornos psiquiátricos (\%) } \\
\hline & & & geral & depressão & ansiedade & esquizofrenia & psicose & t. personalidade & Dep. álcool \\
\hline $\begin{array}{l}\text { Hackett et al., } \\
\text { 1998; Índia }\end{array}$ & $\begin{array}{l}26 \text { PE } 1377 \\
\text { PSE / CID-I0 }\end{array}$ & $\begin{array}{l}\text { População geral } \\
\text { (inquérito) } \\
\text { Crianças 8-12 anos }\end{array}$ & $23,1 / 8,1^{\circ}$ & $\mathrm{NE}$ & $\mathrm{NE}$ & NE & $\mathrm{NE}$ & $\mathrm{NE}$ & $\mathrm{NE}$ \\
\hline $\begin{array}{l}\text { Davies et al., } \\
\text { 2003; R. Unido }\end{array}$ & $\begin{array}{l}67 \text { PE } 47 \mathrm{c} / \\
\text { diabetes } 10249 \\
10202 \text { PSE / } \\
\text { DSM-IV }\end{array}$ & $\begin{array}{l}\text { População geral } \\
\text { (inquérito) } \\
\text { Crianças 5-15 anos }\end{array}$ & $37 / 11 / 9^{\circ}$ & $\mathrm{NE}$ & $\mathrm{NE}$ & NE & $\mathrm{NE}$ & $\mathrm{NE}$ & $\mathrm{NE}$ \\
\hline $\begin{array}{l}\text { Gaitatzis et al., } \\
\text { 2004; R. Unido }\end{array}$ & 5834 PE / CID-9 & $\begin{array}{l}\text { Pacientes de } \\
\text { Banco de dados } \\
\text { /Todas idades }\end{array}$ & $41 \mathrm{em} \mathrm{PE}^{\mathrm{a}}$ & $18,2 / 9,2^{\mathrm{a}}$ & $11,1 / 5,6^{\mathrm{a}}$ & $0,7 / 0,1^{\mathrm{a}}$ & $9 / 2^{\mathrm{a}}$ & $\mathrm{NE}$ & $2,4 / 0,4^{\mathrm{a}}$ \\
\hline $\begin{array}{l}\text { Kobau et al., } \\
\text { 2006; EUA }\end{array}$ & $\begin{array}{l}69 \text { PE } 4154 \\
\text { PSE/Self-reported }\end{array}$ & $\begin{array}{l}\text { População geral } \\
\text { (inquérito) } \\
\text { Crianças e adultos }\end{array}$ & $\mathrm{NE}$ & $\begin{array}{l}39,7^{\mathrm{a}} \\
/ 15,5^{\mathrm{b}}\end{array}$ & $\begin{array}{l}14,9^{\mathrm{b}} \\
/ 6,8^{\mathrm{a}}\end{array}$ & NE & $\mathrm{NE}$ & $\mathrm{NE}$ & $\mathrm{NE}$ \\
\hline $\begin{array}{l}\text { Tellez-Zenteno } \\
\text { et al al., 2007; } \\
\text { Canadá }\end{array}$ & $\begin{array}{l}253 \text { PE, } 36731 \\
\text { PSE / CIDI }\end{array}$ & $\begin{array}{l}\text { População geral } \\
\text { (inquérito) } \geq 15 \text { anos }\end{array}$ & $23,5 / 10,9^{b}$ & $17,4 / 10,8^{b}$ & $12,8 / 4,7^{b}$ & $\mathrm{NE}$ & $\mathrm{NE}$ & $\mathrm{NE}$ & $\mathrm{NE}$ \\
\hline
\end{tabular}


Cockerell et al., 1996, ${ }^{2}$ estudaram transtornos psicológicos agudos na população geral, por meio da "Unidade de Vigilância Neurológica britânica”. Na sua revisão, eles informam que as estimativas relacionadas variam de 2 e $25 \%$ (todos de pacientes oriundos de clínicas). No caso do trabalho dos autores, foram averiguados 64 casos (entre 17 e 69 anos de idade): 48\%, ictal ou pós-ictal; 52\%, interictal. Isso os levou a considerar os transtornos psicológicos agudos infreqüentes tendo em vista a grande população de referência. Dezenove foram considerados transtornos psicológicos agudos relacionados a drogas antiepilépticas (DAE), sendo que 12, à vigabatrina, apesar da estimativa ser imprecisa, segundo os próprios autores por não ser um estudo de incidência.

Mencionamos também os instrumentos diagnósticos mais freqüentemente usados. As comorbidades psiquiátricas são bem descritas em sistemas atuais de classificação, como o DSM-IV e CID-10, embora, como Krishnamoorthy et al., 2007, ${ }^{18}$ lembram, há transtornos psiquiátricos específicos da epilepsia que freqüentemente não são reconhecidos, e não estão cobertos por sistemas atuais de classificação de transtornos psiquiátricos ou de epilepsia, como: consciência alterada, confusão, desorientação, perturbações de memória, ansiedade, disforia, alucinações e síndromes paranóicas; deficiência orgânica cognitiva inclusive queixas de memória; psicoses de epilepsia; transtornos somatoformes afetivos; transtornos de personalidade; ansiedade e fobias específicas da epilepsia; além de problemas relacionados à terapia com DAE e mudanças no EEG. Em conclusão, há uma deficiência geral, em estudos de população com epilepsia selecionada ou não, em diferenciar entre psicopatologia genérica e psicopatologia específica de epilepsia. Matsuura et al., 2003, ${ }^{16}$ lembram que o uso "orgânico" ou "devido a condição médica geral" do DSM-IV ou CID-10 torna difícil de especificar a subcategorização dos transtornos psiquiátricos associados à epilepsia. Para evitar a pobreza das classificações da psicopatologia nos PE, esses últimos autores desenvolveram um esquema penta-axial baseado na classificação da ILAE de Síndromes epilépticas de 1989, para o diagnóstico das síndromes epilépticas, e no CID-10, para os transtornos psiquiátricos, assim composto: eixo 1, variáveis da epilepsia; eixo 2, variáveis do EEG; eixo 3, psicopatologia; eixo 4, transtornos de personalidade; eixo 5, base orgânica.

\section{FATORES DE RISCO E RELAÇÃO TEMPORAL}

As associações entre as manifestações psiquiátricas com a epilepsia poderiam ser espúrias, ou por compartilhar ou contribuir com mecanismos genéticos e/ou ambientais. Isso nos reporta à questão conceitual inicialmente apresentada. Dificilmente poderíamos enquadrar essas comorbidades psiquiátricas de PE em conceito de categorias excludentes. Esse é também o problema das comorbidades psiquiátricas per se, pois como comentam Nierenberg e Sonino, 2004, ${ }^{19}$ no seu artigo em homenagem ao cunhador dos termos comorbidade e clinimetria Alvan Feinstein “... comorbidade psiquiátrica de acordo com padrões do DSM normalmente consiste em transtornos categóricos proximamente relacionados que podem fazer parte do desenvolvimento do transtorno de interesse, por exemplo, fobia social que precede depressão principal. Assim, nós estamos olhando para verdadeiras condições de comorbidade em psiquiatria ou ao invés examinando a inter-relação de sintomas psiquiátricos e carga total psicopatológica?". Gaitatzis et al., 2004, ${ }^{5}$ expressam esta mesma impressão de comorbidade com etiologia compartilhada: a depressão poderia ser facilitada por uma causa comum também à epilepsia, sendo que a psicose poderia ter uma causa comum ou ser conseqüência das crises epilépticas. Além disso, a PE poderia desenvolver depressão pelo estresse da sua vida. Segundo a concepção não ortodoxa de alguns autores e seguindo a segunda definição de comorbidade apresentada no início deste artigo, a comorbidade com epilepsia pode não somente co-acontecer, mas também preceder ou seguir o diagnóstico de epilepsia. A relação temporal de condições de comorbidade é importante para se entender associações causais e mecanismos comuns de doença. Apresentamos a seguir estudos deste tipo. A tentativa de suicídio como sintoma de depressão (razão de chance de 3,9, IC de 1,4$11,5)$ foi identificada como o sintoma de maior risco para CE não provocadas, em estudo a partir de um sistema de vigilância nacional islandês com 324 casos e 647 controles com no mínimo 10 anos de idade. ${ }^{20}$ Outro estudo também liderado por Hesdorffer et al., 2004, ${ }^{21}$ igualmente de casocontrole de base populacional, de 109 casos e 218 controles de 3 a 16 anos, avalia o TDAH neste risco. Segundo esse estudo, entre crianças, uma história de TDAH, predominantemente do tipo desatento, é associada com um risco aumentado para epilepsia, e CE não provocadas ocorrem, mais freqüentemente do que o esperado: razão de chance de 3,7, IC de 1,1-12,8. O mecanismo do TDAH tipo desatento pode compartilhar um antecedente subjacente comum com CE não provocadas: uma ligação hipotética para déficits no sistema de norepinefrina central, segundo Hesdorffer et al., 2004. ${ }^{21}$ Segundo o estudo de Austin et al., 2001,22 em média, as crianças tiveram escores do Child behavior checklist (CBCL) total mais alto e problemas comportamentais internalizantes (por exemplo, ansiedade e depressão) todas às vezes ao experimentar CE periódicas do que ao não experimentá-las (problemas totais, $\mathrm{p}=0,041$ ), com controle variáveis demográficas e das DAE. Os irmãos tiveram escores significativamente mais baixos de problemas de internalização do que tanto as crianças que experimentam (problemas totais ajustados para covariáveis, $\mathrm{p}=0,0001$ ) quanto as que não tinham CE periódicas ( $p=0,0004)$. Os escores de problemas comportamentais externalizantes (por exemplo, TDAH, transtornos de comportamento) não 
foram significativamente diferentes entre crianças com CE ocorrendo ou não periodicamente, e irmãos. CE periódicas predisseram significativa e precocemente problemas de comportamento no curso da epilepsia, até mesmo quando a criança chave, variáveis demográficas e de CE eram controladas. Explicações para esses resultados incluem as possibilidades de que: $\mathrm{CE}$ e problemas de comportamento são causados por um transtorno neurológico subjacente; $\mathrm{CE}$ per se alteram comportamento; as crianças têm respostas negativas psicológicas para a ocorrência das CE. Gaitatzis et al., 2004, ${ }^{3}$ lembram que essa comorbididade parece ser relacionado com fatores endógenos e exógenos (inclusive iatrogênicos) e com a gravidade e cronicidade da epilepsia. Os resultados dos estudos sobre os fatores de risco dessas comorbidades psiquiátricas vêm tendo resultados controversos, mas devem ter origem multifatorial. Schmitz et al., 1999,23 avaliaram os fatores de risco social e biológico para esquizofrenia e depressão em epilepsia em estudo de caso-controle: $25 \mathrm{PE}$ com psicose paranóide-alucinatória, 25 PE com depressão maior, além de 50 PE não-psiquiátricos. Eles concluíram que pacientes esquizofrênicos tiveram uma idade mais precoce de início da epilepsia e uma epilepsia mais grave caracterizada por: história de estado de mal epiléptico, tipos múltiplos de CE (três ou mais) e gravidade das $\mathrm{CE}$, em comparação aos controles não psiquiátricos; sintomas simples de CE eram frequentemente vegetativos e os registros de EEG mostraram várias anormalidades, inclusive descargas nos lobos temporais, mas nenhum lateralização. Esses pacientes tinham antecedente familiar do transtorno, falta de relações interpessoais, dependência social e fracasso profissional. Em relação aos pacientes deprimidos, eles eram: significativamente mais velhos que os controles não psiquiátricos e freqüentemente sofreram mais de epilepsias focais do lobo temporal; não diferiram dos controles em relação à gravidade da epilepsia; não havia nenhuma variável psicossocial significativamente vinculada à depressão. No entanto, é controverso o papel do lobo temporal na gênese da depressão.

Schmitz et al., $1999,{ }^{23}$ a partir de estudo casocontrole de base clínica, concluíram que PE com psicose esquizofrênica tinham epilepsia mais grave, enquanto os depressivos sofriam mais de epilepsia focal do lobo temporal, ambos comparativamente aos controles, PE sem comorbidade psiquiátrica. Matsuura et al., 2003, ${ }^{16} \mathrm{em}$ um estudo multicêntrico sem grupo controle, concluíram que havia relação entre comorbidade psiquiátrica e retardo mental, epilepsia do lobo temporal e freqüência alta de CE. Swinkels et al., 2001,10 em estudo comparativo com dados clínicos e populacionais, inferiram que prevalência de transtornos de humor tende a ser um pouco mais alta para os PE do lobo temporal do que dos extratemporal, especialmente na prevalência do último ano, mas a diferença não é estatisticamente significativa.
Outra questão é o papel das DAE nas mencionadas comorbidades. Schmitz et al., 1999, ${ }^{23}$ acharam: uma ligação inversa entre valproato e depressão, mas não entre carbamazepina e depressão; nenhuma confirmação de uma ligação entre tratamento com primidona ou fenobarbital e depressão. Nesse estudo, o papel das DAE está menos claro para psicose esquizofreniforme, mas politerapia não foi vinculada à depressão, mas sim com psicose esquizofreniforme. Matsurura et al., 2003,16 com o estudo de 398 pacientes com epilepsia, mas sem grupo controle, e a partir de análise de regressão logística, concluíram que existem três fatores de risco para o desenvolvimento de transtornos psiquiátricos nos mencionados pacientes: retardo mental, epilepsia do lobo temporal, especialmente a com alta freqüência de crises (transtornos não psicóticos), e alta freqüência de CE.

\section{Comorbidade e qualidade de vida}

QVLS representa um conceito subjetivo, com uma perspectiva multidimensional que atinge o funcionamento físico, emocional e social. ${ }^{1}$ Maia Filho et al., 2004,24 lembram que na dimensão psicológica da QVLS da infância influenciam as comorbidades psiquiátricas, além de sentimentos, comportamentos, afetividade, medos, autoestima, felicidade e planos/objetivos. Strine et al., 2005,13 mencionam que vários estudos sugerem que a QVLS em PE é mais fortemente associada com estados de humor do que com freqüência de CE ou a sua gravidade. QVLS é uma medida de resultado que crescentemente está sendo usada para avaliar efeitos (outcomes) em estudos clínicos de pacientes com doenças crônicas. Uma distinção deve ser feita entre doenças crônicas simples e complexas (condições que envolvem doenças múltiplas que requerem a atenção de vários provedores de cuidados de saúde ou instalações e possivelmente cuidados domiciliares ou comunitários), como pelo menos parte dos PE. ${ }^{1}$ Fatores adicionais a serem considerados quando se define multimorbidade inclui a gravidade das condições ${ }^{1}$. Outra limitação metodológica da maioria dos estudos avaliados sobre QVLS foi o seu fracasso em considerar a influência da comorbidade psiquiátrica. ${ }^{1}$ A QVLS tende a diminuir com a idade, ao contrário do número de diagnósticos. Assim, é apropriado considerar a idade como uma variável confundidora em potencial. ${ }^{1}$ Os resultados foram contraditórios em relação ao gênero, e pouco foi informado sobre os efeitos de outras variáveis confundidoras (por exemplo, dados sociodemográficos e econômicos, hábitos de saúde, apoio social, número de drogas prescritas). ${ }^{1}$ No entanto, o número de comorbididades era o preditor independente mais forte de QVLS. ${ }^{1}$ A revisão de Katon et al., 2007, ${ }^{25}$ sobre pacientes com doenças crônicas descreve interações próximas e complexas entre comorbidade somática, psiquiátrica (depressão e ansiedade) e medidas fisiológicas. Essa revisão 
achou que os pacientes com doença crônica associada a depressão ou ansiedade, comparados aos com doença crônica isolada tinham números significativamente mais altos de sintomas médicos após controle da gravidade da doença. Mais especificamente, Meldolesi et al., 2006, ${ }^{26}$ objetivaram elucidar a associação entre QVLS e fatores sociodemográficos, fatores clínicos de CE, depressão e ansiedade em 106 PE farmacorresistente do lobo temporal. A depressão foi constantemente o preditor mais forte de escores mais baixos em quase todos domínios de QVLS. Também, mas secundariamente, a gravidade de sintomas de ansiedade era significativamente associada com escores mais baixos em vários domínios de QVLS. Enquanto as variáveis das CE tiveram uma associação mais fraca com QVLS, a ausência de pacientes sem crises pode ter obscurecido uma relação entre freqüência de CE e QVLS, segundo os autores. ${ }^{26}$ Outra questão é o tipo de comorbidade e a sua repercussão. Mathers et al., 2006, apud. Wiebe e Herdorffer, 2007, 27 avaliaram o impacto relativo de vários tipos de comorbidade em QVLS: por exemplo, estresse pós-traumático e depressão têm o impacto mais alto em QVLS, mas esquizofrenia tem impacto mais baixo.

\section{Índices de multimorbidade: avaliação de qualidade de vida, mortalidade e custos}

Foram desenvolvidos índices de multimorbidade para avaliar o impacto de uma constelação de condições de comorbidades em uma variedade de indicadores de saúde: QVLS, uso de recursos de saúde e sobrevida. ${ }^{28}$ Medidas de multimorbidade são importantes em estudos epidemiológicos e de serviços de saúde. No entanto, um único índice não pode predizer uma variedade de efeitos, mas deve ser usado para predizer um resultado específico de interesse. Assim, evidências sugerem que QVLS é prevista com maior precisão por índices de comorbidade que incorporam medidas de gravidade. Fortin et al., 2005, avaliam três índices de multimorbidade: Cumulative Illness Rating Scale (CIRS), Charlson index (Charlson) e Functional Comorbidity Index (FCI). Os dois primeiros são dos mais válidos e confiáveis para avaliação de multimorbidade. ${ }^{28}$ No entanto, o primeiro foi considerado adequado quando o efeito de interesse de avaliação é a QVLS. ${ }^{28}$ Isso pode ser devido ao fato que o CIRS avalia o número e a gravidade de todas as doenças crônicas, mas o FCI avalia um número limitado de diagnósticos e não leva em consideração a gravidade da doença. Um único estudo foi recuperado com o uso em epilepsia de um dos três índices já mencionados, o de Lee et al., 2005,,$^{29}$ sobre 549 PE com epilepsia focal farmacorresistente à monoterapia inicial com DAE. Esse é um estudo retrospectivo feito a partir de dados administrativos sobre o efeito da comorbidade nas internações e custos. As probabilidades (odds) de hospitalização eram 3,7 vezes maiores entre pacientes com comorbidades do que para os sem (razão de chance $=3,7$, $95 \%$ IC $=1,7-7,9)$, enquanto o custo do tratamento para todo cuidado médico era $136 \%$ mais alto $(\mathrm{P}<0,05)$. Entre todos os pacientes, "convulsões não especificadas" era o sintoma mais geralmente informado (57,4\%), e cefaléia $(10,6 \%)$, hipertensão (10,0\%) e depressão $(6,9 \%)$ eram as comorbidades freqüentemente registradas. Os autores concluem que depressão se revelou um preditor importante de custos de cuidado médico (por exemplo, 83,1\% maiores custos do que os pacientes sem depressão) e na probabilidade de hospitalização (razão de chance $=3,5,95 \% \mathrm{IC}=2,13-5,8$ ), entre todas as condições de comorbidade.

\section{Repercussão na prática clínica}

É possível que certas comorbidades da epilepsia compartilhem uma etiologia subjacente e o seu tratamento reduzirá a gravidade da epilepsia, como foi proposto por alguns investigadores no caso de transtornos do humor e ansiedade simultâneos com epilepsia. Reciprocamente, tratamento adequado das CE pode reduzir a gravidade das mencionadas comorbidades. ${ }^{27}$ No entanto, a comorbidade psiquiátrica nas $\mathrm{PE}$ freqüentemente exige o uso de drogas psicotrópicas associadas às DAE, mas devido ao receio do eventual risco da indução de CE, é comum o clínico postergar a sua indicação. ${ }^{4}$

De modo geral, há carência de estratégias evidênciabaseadas relativas ao tratamento de pacientes com comorbidades, pois tipicamente elas focam transtornos isolados e não se discute a sua propriedade para os com comorbidades, segundo Wiebe e Hesdorffer, 2007. ${ }^{27}$ Freqüentemente, em ensaios clínicos, os pacientes com condições de comorbidades importantes são excluídos. ${ }^{27}$ As necessidades de serviço de saúde mental poderiam ser consideráveis nessa população, com uma carga não revelada significante de comorbidade psiquiátrica. Embora a estratificação e análise de subgrupos para variáveis de prognóstico sejam comuns em ensaios clínicos, poucos valorizam a interação entre comorbidades e a intervenção. ${ }^{27}$ Em relação aos fatores de risco presumidos ou conhecidos para epilepsia, profissionais de saúde deveriam estar atentos para o significado da saúde mental dos pacientes e o seu papel na QVLS, e, por conseguinte, a adoção de uma abordagem biopsicossocial poderia ser mais útil para atender as necessidades dos pacientes, do que apenas uma única de base orgânica.

Maia Filho et al., 2006,17 enfatizam que atualmente a abordagem clínica da criança com epilepsia ultrapassa o simples controle das crises epilépticas, devendo haver atenção especial ao adequado diagnóstico e tratamento das comorbidades psiquiátricas a fim de melhorar a sua qualidade de vida. 


\section{DISCUSSÃO/CONCLUSÕES}

Reconhece-se que as comorbidades psiquiátricas são mais prevalentes nas PE do que na população geral, sendo a depressão, ansiedade, psicoses, esquizofrenia, transtornos de personalidade e dependência alcoólica as mais comuns. As crianças também são acometidas. Dificuldades emocionais, comportamentais e de relação são comuns em crianças com epilepsia, mais do que nas com diabetes, por exemplo, e elas são mais freqüentes nos com epilepsia complicada. Apesar das taxas maiores do que a população geral em todas as idades, quanto mais velho, menor a probabilidade da maioria dessas comorbidades psiquiátricas, especialmente os transtornos do humor (em mulheres há tendência ao decréscimo, mas em homens os níveis parecem ficar estáveis), ansiedade e transtornos relacionados ao uso de substâncias psicoativas, mas, possivelmente, transtornos do pânico e agorafobia tendem a aumentar. Outros dados sobre distribuição sócio-demográfica levam a crer que as $\mathrm{PE}$ com as comorbidades psiquiátricas tendem a ter níveis mais baixos de educação e níveis mais altos de desemprego. Essas comorbididade parecem ser relacionadas com fatores endógenos e exógenos (iatrogênicos e ambientais), com a gravidade e cronicidade da epilepsia, sendo principalmente vinculadas à epilepsia farmacorresistente e a do lobo temporal, principalmente a farmacorresistente. Quanto à classificação dessas comorbidades, os estudos usualmente não costumam diferenciar a psicopatologia genérica da relacionada à epilepsia. Para pacientes refratários à monoterapia inicial com DAE, a presença de comorbidades, especialmente da depressão, parece estar associada com um aumento significativo nos custos dos cuidados médicos. A comorbidade psiquiátrica afeta ambos aspectos da QVLS, físicos e psicossociais. Embora haja compreensão dos mencionados aspectos epidemiológicos, apesar da diversidade metodológica dos estudos, pouco é conhecido sobre os fatores causais e mecanismos básicos dessas comorbidades, intervenções clínicas para prevenilas, ou a abordagem de pacientes com comorbidades. Será importante avaliar o efeito de intervenções para epilepsia na comorbidade e também o efeito de intervenções nas comorbidades em efeitos fundamentais da epilepsia, como as crises epilépticas, QVLS, mortalidade e, também, custos do atendimento.

\section{REFERÊNCIAS}

1. Fortin M, Lapointe L, Hudon C, Vanasse A, Ntetu AL, Maltais D. Multimorbidity and quality of life in primary care: a systematic review. Health Qual Life Outcomes. 2004;2:51.

2. Cockerell OC, Moriarty J, Trimble M, Sander JW, Shorvon SD. Acute psychological disorders in patients with epilepsy: a nation-wide study. Epilepsy Res. 1996;25(2):119-31.

3. Gaitatzis A, Trimble MR, Sander JW. The psychiatric comorbidity of epilepsy. Acta Neurol Scand. 2004;110:207-20.
4. Fiszman A, Gomes MM. A interface entre epilepsia e psiquiatria. Brazilian J Epilepsy Clin Neurophysiol. 2001;7(2):55-67.

5. Gaitatzis A, Carroll K, Majeed A, Sander W. The epidemiology of the comorbidity of epilepsy in the general population. Epilepsia. 2004;45:1613-22.

6. Tellez-Zenteno JF, Patten SB, Jetté N, Williams J, Wiebe S. Psychiatric comorbidity in epilepsy: a population-based analysis. Epilepsia. 2007;48(12):2336-44.

7. Maia Filho HS Gomes MM, Araújo APQC. Associação entre epilepsia e enxaqueca: uma revisão crítica. Brazilian J Epilepsy Clin Neurophysiol. 1999;5(4):131-4.

8. Nuyen J, Schellevis FG, Satariano WA, Spreeuwenberg PM, Birkner MD, van den Bos GA, Groenewegen PP. Comorbidity was associated with neurologic and psychiatric diseases: a general practice-based controlled study. J Clin Epidemiol. 2006;59:1274-84.

9. Kobau R, Gilliam F, Thurman DJ. Prevalence of self-reported epilepsy or seizure disorder and its associations with self-reported depression and anxiety: results from the 2004 HealthStyles Survey. Epilepsia. 2006;47(11):1915-21.

10. Swinkels WA, Kuyk J, de Graaf EH, van Dyck R, Spinhoven P. Prevalência of Psychopathology in Dutch Epilepsy Inpatients: A Comparative Study. Epilepsy Behav. 2001;2(5):441-447.

11. Hackett R, Hackett L, Bhakta P. Psychiatric disorder and cognitive function in children with epilepsy in Kerala, South India. Seizure. 1998; $7(4): 321-4$.

12. Davies S, Heyman I, Goodman R. A population survey of mental health problems in children with epilepsy. Dev Med Child Neurol. 2003;45(5):292-5.

13. Strine TW, Kobau R, Chapman DP, Thurman DJ, Price P, Balluz LS. Psychological distress, comorbidities, and health behaviors among U.S. adults with seizures: results from the 2002 National Health Interview Survey. Epilepsia. 2005;46(7):1133-9.

14. Stefansson SB, Olafsson E, Hauser WA. Psychiatric morbidity in epilepsy: a case controlled study of adults receiving disability benefits. J Neurol Neurosurg Psychiatry. 1998;64(2):238-41.

15. Gomes MM, Kropf LA, Beeck ES, Figueira IL. Inferences from a community study about non-epileptic events. Arq Neuropsiquiatr. 2002;60(3-B):712-6.

16. Matsuura M, Oana Y, Kato M, Kawana A, Kan R, Kubota H, Nakano T, Hara T, Horikawa N.A multicenter study on the prevalência of psychiatric disorders among new referrals for epilepsy in Japan. Epilepsia. 2003;44(1):107-14.

17. Maia Filho HS, Costa CRM, Gomes MM. Epilepsia e saúde mental na infância. Journal of epilepsy and clinical neurophysiology. 2006;12(2):79-88.

18. Krishnamoorthy ES, Trimble MR, Blumer D. The classification of neuropsychiatric disorders in epilepsy: A proposal by the ILAE Commission on Psychobiology of Epilepsy. Epilepsy Behav. 2007;10:349-53.

19. Nierenberg AA, Sonino N. De clinical observations to clinimetrics: a tribute to Alvan R. Feinstein, MD. Psychother Psychosom. 2004;73(3):131-3.

20. Hesdorffer DC, HauserWA, Olafsson E, Ludvigsson P, Kjartansson O. Depression and suicide attempt as risk factors for incident unprov-ed seizures. Ann Neurol. 2006;59:35-41.

21. Hesdorffer DC, Ludvigsson P, Olafsson E, Gudmundsson G, Kjartansson $\mathrm{O}$, Hauser WA. ADHD as a risk factor for incident unprov-ed seizures and epilepsy in children. Arch Gen Psychiatry. 2004;61: 731-736.

22. Austin JK, Dunn DW, Caffrey HM, Perkins SM, Harezlak J, Rose DF. Recurrent seizures and behavior problems in children with first recognized seizures: a prospective study. Epilepsia. 2002;43(12): 1564-73. 
23. Schmitz EB, Robertson MM, Trimble MR. Depression and schizophrenia in epilepsy: social and biological risk factors. Epilepsy Res. 1999;35(1):59-68.

24. Maia Filho HS Gomes MM. Epilepsia na infância e qualidade de vida. J Epilepsy Clin Neurophysiol 1999;5(4):131-4.

25. Katon W, Lin EH, Kroenke K. The association of depression and anxiety with medical symptom burden in patients with chronic medical illness. Gen Hosp Psychiatry. 2007;29(2):147-55.

26. Meldolesi GN, Picardi A, Quarato PP, Grammaldo LG, Espósito V, Mascia A, Sparano A, Morosini P, Di Gennaro G. Factors associated with generic and disease-specific quality of life in temporal lobe epilepsy. Epilepsy Research. 2006;69:135-46.

27. Wiebe S, Hesdorffer DC. Epilepsy: being ill in more ways than one. Epilepsy Curr. 2007;7(6):145-8.
28. Fortin M, Hudon C, Dubois MF, Almirall J, Lapointe L, Soubhi H. Comparative assessment of three different indices of multimorbidity for studies on health-related quality of life. Health Qual Life Outcomes. 2005;3:74.

29. Lee WC, Arcona S, Thomas SK, Wang Q, Hoffmann MS, Pashos CL. Effect of comorbidities on medical care use and cost among refractory patients with partial seizure disorder. Epilepsy Behav. 2005;7(1):123-6.

Endereço para correspondência:

Marleide da Mota Gomes

Instituto de Neurologia da UFRJ - Programa de Epilepsia

Av. Venceslau Braz, 95 - Botafogo

CEP 22290-140, Rio de Janeiro, RJ, Brasil 